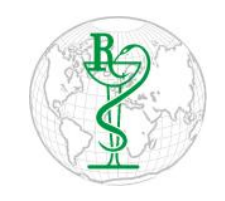

INDO GLOBAL JOURNAL OF

PHARMACEUTICAL SCIENCES

ISSN 2249- 1023

\title{
Homology Modelling, Phylogenetic Analysis, and Molecular Docking of Glutamine Aminotransferase, gatD from Clostridium botulinum
}

\author{
Praveen Kumar ${ }^{1 *}$, Archana G. Mohanan ${ }^{2}$, Anil Kumar AK ${ }^{1}$ \\ ${ }^{1}$ Govt. College for Women, Thiruvananthapuram, Kerala, India, 695014 \\ ${ }^{2}$ Rajiv Gandhi Centre for Biotechnology, Thiruvananthapuram, Kerala, India
}

Address for Correspondence: Praveen Kumar, praveen@gcwtvm.ac.in

\begin{tabular}{l} 
Received: \\
19.04.2020 \\
Accepted: \\
04.10 .2020 \\
Published: \\
20.12 .2020 \\
\\
Keywords \\
gatD, Amidation, \\
Molecular \\
docking, \\
Autodock vina, \\
Clostridium \\
botulinum. \\
\hline
\end{tabular}

ABSTRACT: Glutamine amidotransferase (gatD) catalyze the amidation of the $\alpha$-carboxyl group of the Disoglutamate residue in Lipid II resulting in the formation of D-isoglutamine. Mutants deficient in Lipid II amidation show a reduced cross-linking of peptidoglycan and are highly susceptible to antibiotics. Thus, interfering with amidation reaction in gram-positive bacteria may represent a useful strategy to combat the pathogens. In the present investigation, gatD from Clostridium botulinum was subjected to homology modelling, phylogenetic analysis and molecular docking. As the crystal structure for gatD was unavailable, the strategy of homology modelling was utilized to generate its tertiary structure. The generated model was refined, energy minimized and validated to ensure the better quality. The phylogenetic analysis revealed the presence of three distinct clusters and highlighted the divergence in gatD polypeptide sequence distributed throughout the gram-positive bacterial species. The ligand binding pockets in gatD and the associated residues were identified using PrankWeb. The results from molecular docking study revealed that Compound 1 (Pubchem CID: 260474), Triciribine, Iodotubercidin, Azasangivamycin, Sangivamycin, Toyocamycin, Tecadenoson, Compound 2 (Pubchem CID: 324510) bind effectively to the ligand binding pocket of gatD with a binding energy of $-8.6,-8.3,-8.1,-8.1,-8.0,-7.8,-7.7,-7.1$ respectively. As majority of these compounds are also known as anti-cancer, antiviral agents, they are worth of further experimental investigations in terms of inhibition of cell wall synthesis, amidation and anti-microbial properties. Computer-aided drug design is useful for the identification of novel drug targets and to design new drugs. The experimental studies have to be carried to evaluate the amidation interfering properties of the predicted compounds. () 2020 iGlobal Research and Publishing Foundation. All rights reserved.

Cite this article as: Kumar, P.; Mohanan, A.G.; Anil, K.A.K. Homology modelling, phylogenetic analysis and molecular docking of glutamine aminotransferase, gatD from Clostridium botulinum. Indo Global J. Pharm. Sci., 2020; 10(4): 46-57. DOI: http://doi.org/10.35652/IGJPS.2020.10408 .

\section{INTRODUCTION}

The bacterial cell wall mainly consists of a peptidoglycan polymer located external to the cytosolic membrane. The peptidoglycan units consist of the disaccharide $\mathrm{N}$ acetylglucosamine and $N$-acetylmuramic acid to which a pentapeptide is attached. The lipid II is a carrier of complete disaccharide-peptide monomer unit. Lipid II is transferred from the cytoplasmic membrane to externally located sites where polymerization of the disaccharide-peptide monomer takes place [1]. The peptide moiety in the lipid II undergoes secondary modifications prior to the polymerization reactions such as amidation, the addition of extra amino acids, and esterification, etc. Recently, the crystal structure of an enzyme, GatD/MurT complex from $S$. aureus was solved at high resolution [2]. Amidation of the $\alpha$-carboxyl group of the D-isoglutamate residue in Lipid II results in the formation of D-isoglutamine [3]. This reaction is catalyzed by GatD/MurT complex [4]. This complex comprises of two proteins, GatD and MurT, which assemble into a binary complex. GatD is homologous to the catalytic domains of glutamine amidotransferases, while MurT is identical to the sequence to the substrate-binding domains of Mur ligases. The amidation of $\alpha$-D-isoglutamic acid of cell wall precursor stem peptides is 


\section{Indo Global Journal of Pharmaceutical Sciences, 2020; 10(4): 46-57}

catalyzed by GatD/MurT complex in an ATP-dependent reaction [2]. Mutants deficient in Lipid II amidation show a reduced cross-linking of peptidoglycan and are highly susceptible to antibiotics. Thus, interfering with amidation reaction in gram-positive bacteria may represent a useful strategy to combat the pathogens.

Clostridium botulinum is a Gram-positive, rod-shaped bacterium, which produces a neurotoxin, botulinum. Four different groups are recognized within $C$. botulinum in which Groups I and II are primarily responsible for human botulism. A dose of 30-100 ng (3000 MLD M0 (mouse minimum lethal doses) of neurotoxin is sufficient to cause human botulism [5]. There are reports confirming the isolation of strains of $C$. botulinum resistance to Penicillin and Metronidazole [6]. Antibiotics could be useful to eliminate $C$. botulinum from the intestine, but this might aggravate botulinum symptoms [7].

Glutamine amidotransferase (gatD) from C. botulinum is not well characterized and the crystal structure of gatD has not yet been elucidated. Computer-aided drug design is useful for the identification of novel drug targets and to design new drugs [8].In this paper, we have explored the possibility of using gatD from $C$. botulinum as a drug target. Further, an attempt was made to study the evolutionary relationship among gatD from gram-positive bacterial species.

\section{MATERIALS AND METHODS}

\section{Phylogenetic Tree construction}

The gatD protein sequences of different gram-positive bacteria were retrieved from Uniprot and the sequences were downloaded in 'fasta' format. The phylogenetic analysis of gatD sequences were carried out using the Molecular Evolutionary Genetic Analysis program version 7.0 [9]. ClustalW algorithm was used for multiple sequence alignment. A neighbour-joining algorithm with 1,000 bootstraps was used in order to infer the evolutionary history. Total sequences involved in the phylogenetic analysis amounted to 47. All positions containing gaps and missing data were eliminated. There were 205 positions in the final dataset.

\section{Homology modelling and validation}

The sequence gatD protein of C.botulinum was retrieved from Uniprot KB (https://www.uniprot.org/). The sequence in 'fasta' format was submitted to swiss-model workspace to generate a 3-D homology-based model of the protein (model1) (https://swissmodel.expasy.org/). The model generated in the swiss-model workspace was submitted to 3-D refine protein structure refinement so as to obtain a refined model (model-2). The model generated in Swiss-model workspace was further subjected to energy minimization in UCSFChimera (version-1.13.1) (model-3). MolProbity score, stereochemical property (Ramachandran Plot) and global QMEAN score of all three models was assessed by Ramachandran plot analysis in the swiss structure assessment web server (https://swissmodel.expasy.org/assess). Assessment of protein models with three-dimensional profiles was carried out in VERIFY 3D (https://servicesn.mbi.ucla.edu/Verify3D/) andoverall quality factor for non-bonded atomic interactions was assessed by ERRAT score (https://servicesn.mbi.ucla.edu/ERRAT/)

\section{Molecular Docking}

The gatD model obtained by homology modelling and energy minimization (model-2) was submitted to PrankWeb (http://prankweb.cz/)for ligand binding site prediction and conservation Exploration. The model-2 was submitted to SPOT-ligand, an online server for virtual screening [10]. Molecular docking was done by using AutodockVina (version-1.5.6) with chimera (version-1.13.1) used as an interface. The energy minimization was performed both for ligand and receptor and mol2 files were generated. The centre grid coordinates in AutodockVina were identified based on the measurement of the pocket-1(in PrankWeb). The coordinates for grids in AutodockVina were $-49 \times 39 \times 37$ while size grids used for $\mathrm{x}, \mathrm{y}, \mathrm{z}$ were $20 \times 20 \times 20$, respectively. The number of binding modes was fixed at 5 . The rest of the parameters were kept in default. Discovery Studio 4.1 visualizer (http: // accelrys.com/products /discovery-studio/) was used for the visualization of docking results of top 8 ligands with gatD.

\section{RESULTS AND DISCUSSION}

\section{Phylogenetic Analysis of gatD}

The phylogenetic analysis of deduced polypeptide sequences of gatD from gram-positive bacteria revealed the presence of two major and one minor cluster (Figure 1). It was found that clusters 1 and 3 were polyphyletic in origin with four and three clades respectively. The cluster I comprised of gatD from Streptooccus, Enterococcus and related bacterial species. The gatD from genus Clostridium and Hathewaya formed a unique cluster (Cluster II) and is unrelated to cluster III. The gatD from Staphylococcus, Streptomyces and related species are included in Cluster III. It was interesting to note that the gatD from C.botulinum is evolutionary related to cluster I. All the sequences within a cluster indicated orthologs from different species of gram-positive bacteria. The ancestral gatD sequence has undergone a series of duplication and diversification event in each cluster. Overall, the phylogenetic 


\section{Indo Global Journal of Pharmaceutical Sciences, 2020; 10(4): 46-57}

tree constructed by the neighbour-joining method indicated distributed throughout the bacterial species.

considerable divergence in gatD polypeptide sequence

Figure 1. Phylogenetic relationships among gatDprotein sequences from gram-positive bacterial species constructed by neighbour-joining algorithm. Theuniport ids are given inparenthesis. The bootstrap values are shown in nodes and are percentages of 1000 replications. The blue coloured upright triangle represents the gatDprotein sequence from $C$. botulinum.

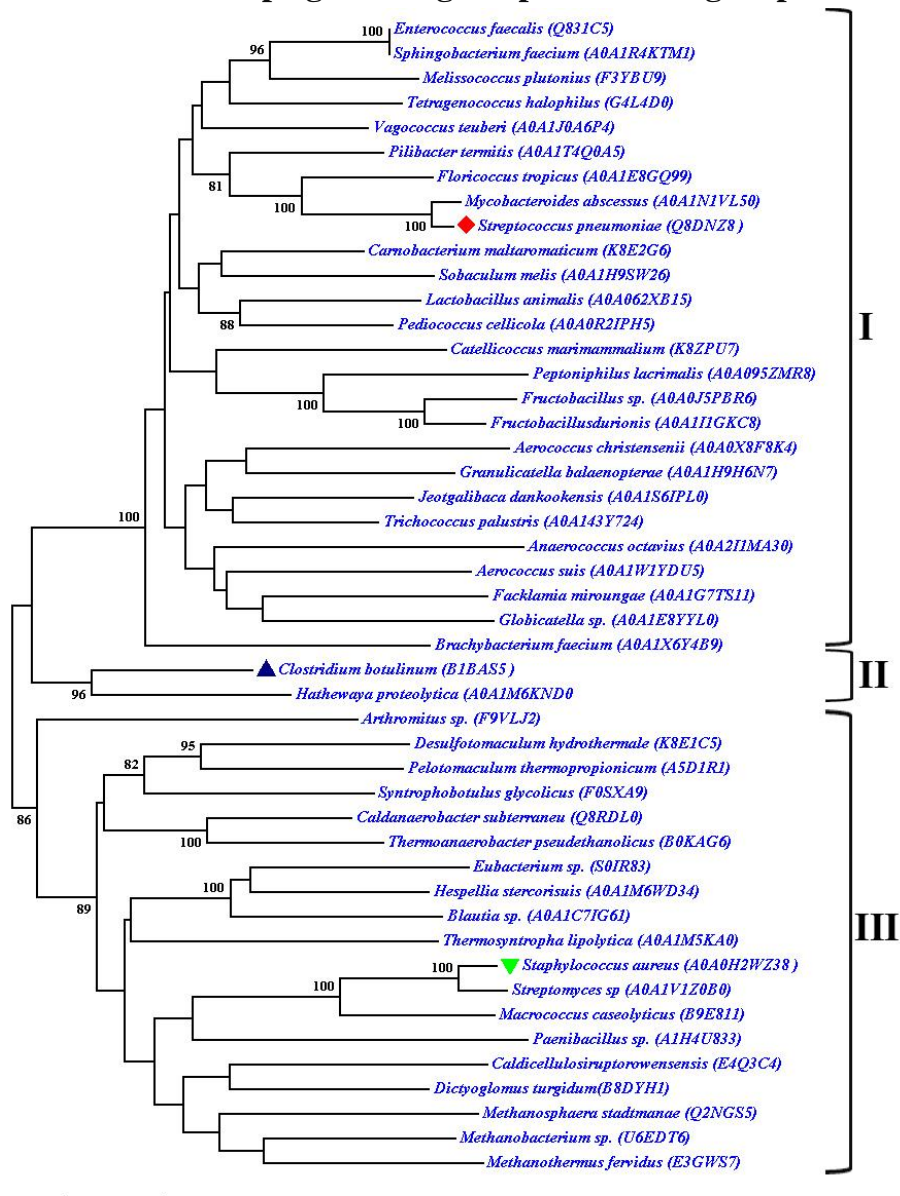

Figure 2. Pairwise alignment of gatD from S. pneumoniae and C. botulinum

08DHZ8_(S. pnezmoniae) B1BAS5 ${ }^{-}$(c. botulinm)

08DHz8_(s. prezmoniae) B1BAS5 (c. botulimm)

Q8DHZ8 (S. pRemoniae) B1BAS5 ${ }^{-}$(c. botulinm)

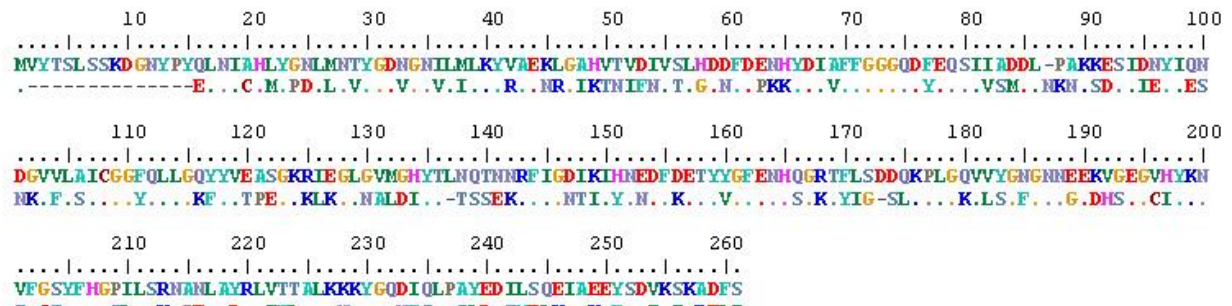

Table 1: Validation of homology- based models of gatD from C. botulinum

\begin{tabular}{cccccc}
\hline Models & $\begin{array}{c}\text { MolP } \\
\text { robity } \\
\text { Score }\end{array}$ & $\begin{array}{c}\text { \% of } \\
\text { Ramachand } \\
\text { ran } \\
\text { favoured } \\
\text { residues }\end{array}$ & $\begin{array}{c}\text { QME } \\
\text { AN } \\
\text { Z- } \\
\text { scores }\end{array}$ & $\begin{array}{c}\text { Veri } \\
\text { fy } \\
\text { scor } \\
\text { e }\end{array}$ & $\begin{array}{c}\text { ERR } \\
\text { AT } \\
\text { score }\end{array}$ \\
\hline Model-1 & 1.73 & 92.7 & -0.51 & $\begin{array}{c}82.9 \\
6\end{array}$ & 97.3 \\
Model-2 & 1.43 & 93.45 & 0.77 & $\begin{array}{c}84.7 \\
5\end{array}$ & 99.5 \\
Model-3 & 2.01 & 95.02 & -0.25 & $\begin{array}{c}83.4 \\
1\end{array}$ & 88.6 \\
\hline
\end{tabular}


Indo Global Journal of Pharmaceutical Sciences, 2020; 10(4): 46-57

Table 2: Prediction of ligand binding pocket in gatD of $C$. botulinum by using PrankWeb

\begin{tabular}{c|c|c|c|c|c|c}
\hline Name & Rank & $\begin{array}{c}\text { Conservation } \\
\text { Score }\end{array}$ & $\begin{array}{c}\text { Coordinate } \\
\text { Center-x }\end{array}$ & $\begin{array}{c}\text { Coordinate } \\
\text { Center-y }\end{array}$ & $\begin{array}{c}\text { Coordinate } \\
\text { Center-z }\end{array}$ & $\begin{array}{c}\text { Amino acid residues associated with } \\
\text { pockets }\end{array}$ \\
\hline Pocket 1 & 1 & 30.3525 & -46.9701 & 38.9523 & 36.7012 & $\begin{array}{c}\text { Tyr103, Val104, Pro106, Thr123, Arg127, } \\
\text { Asn148, Val14, His149, Ser150, Gly151, } \\
\text { Tyr15, Tyr188, His190, Gly58, Gly59, } \\
\text { Gln60, Asp61, Gly64, Cys93, Gly94, Gln97 }\end{array}$ \\
\hline Pocket 2 & 2 & 1.4414 & -44.5581 & 55.7616 & 33.3535 & $\begin{array}{c}\text { Thr153, Ile155, Asn171, Glu173, His175, } \\
\text { Ser176 }\end{array}$ \\
\hline Pocket 3 & 3 & 0.9354 & -57.0919 & 60.2349 & 44.3219 & $\begin{array}{c}\text { Asp138, Phe140, Ile180, Tyr181, Lys182, } \\
\text { Asn183 }\end{array}$ \\
\hline
\end{tabular}

Table 3: Table showing list of compounds, molecular weight, autodock vina binding energy, number of H-bonds formed and residues in the pocket involved in $\mathbf{H}$-bonding.

\begin{tabular}{l|c|c|c|c}
\hline \multicolumn{1}{c|}{ Ligands } & $\begin{array}{c}\text { Molecular } \\
\text { weight } \mathbf{( g / m o l )}\end{array}$ & $\begin{array}{c}\text { Autodockvina binding } \\
\text { energy (kcal/mol) }\end{array}$ & $\begin{array}{c}\text { No. of H- } \\
\text { bond formed }\end{array}$ & Residues in pocket involved in H-bonding \\
\hline $\begin{array}{l}\text { Compound 1 } \\
\text { (Pubchem CID: } \\
\text { 260474) }\end{array}$ & 325.28 & -8.6 & 6 & Gly59, Asp61, Gln64, Gly94, Arg127, Ser150 \\
\hline Triciribine & 320.3 & -8.3 & 3 & Asp61, Ser150, His190 \\
\hline Iodotubercidin & 392.15 & -8.1 & 5 & Gly59, Asp61, Gln64, Gln97, Asn128 \\
\hline Azasangivamycin & 310.27 & -8.1 & 5 & Gly59, Gln64, Gln97, Asn148, His190 \\
\hline Sangivamycin & 309.28 & -8 & 3 & Gln64, Gln97, Asn148 \\
\hline Toyocamycin & 291.26 & -7.8 & 3 & Gly59, Gln97, His190 \\
\hline Tecadenoson & 337.33 & -7.7 & 3 & Gly59, Gln97, His190 \\
\hline $\begin{array}{l}\text { Compound 2 } \\
\text { (Pubchem CID: }\end{array}$ & 295.29 & -7.1 & & 5 \\
324510) & & & & \\
\hline
\end{tabular}

Figure 3. Ramachandran plot of gatD from C. botunilum. A:-Homology models generated by Swiss-model (model-1), B:- model obtained by energy minimization in Chimera (model-2) and C:- model refined by 3-D refine (model-3).
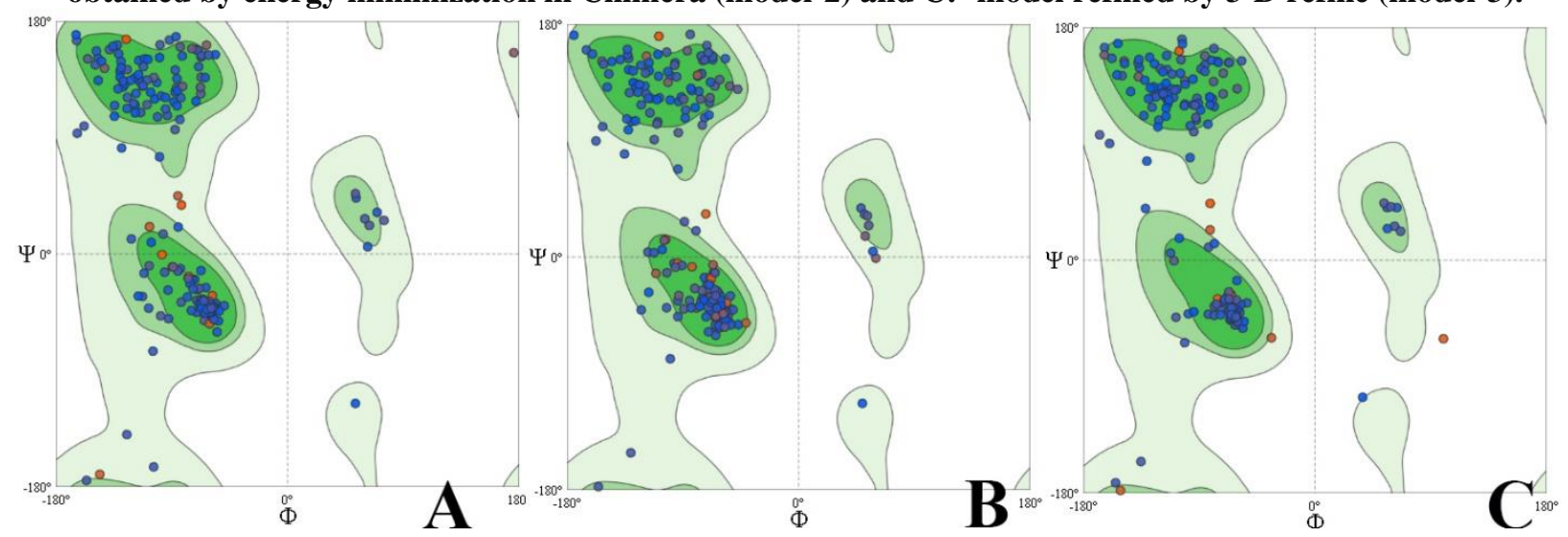
Indo Global Journal of Pharmaceutical Sciences, 2020; 10(4): 46-57

Table 4. Chemical structure and IUPAC names of the compounds used for molecular docking

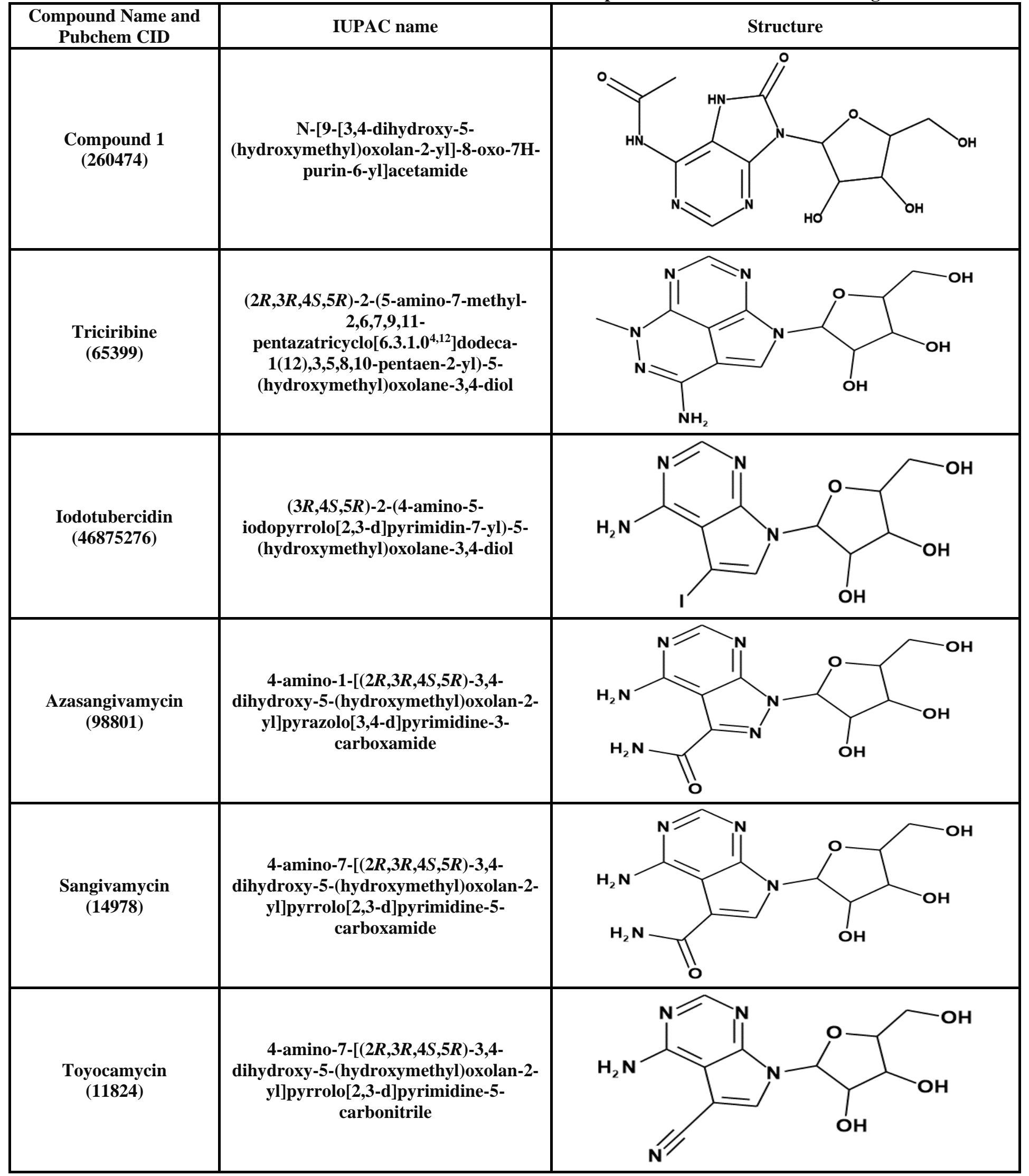


Indo Global Journal of Pharmaceutical Sciences, 2020; 10(4): 46-57

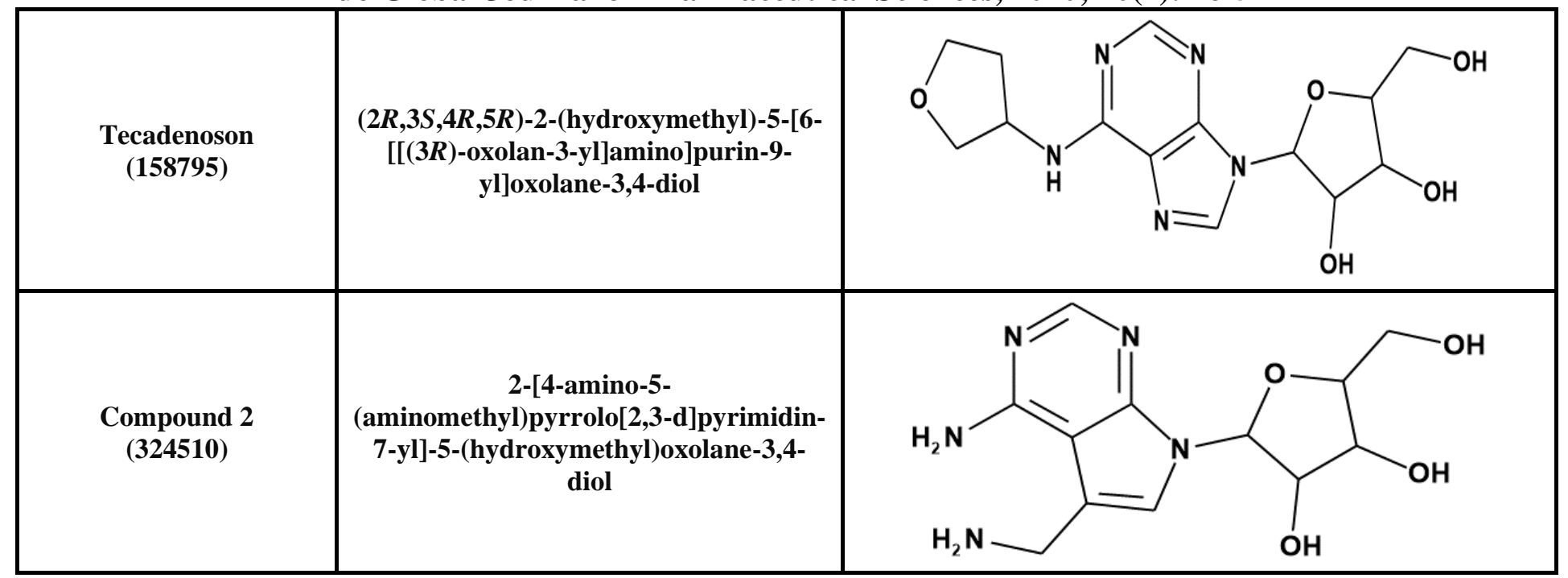

Figure 4. Structure comparison of gatD from $C$.

botunilum: Homology models generated by Swissmodel(blue), model refined by 3-D refine (red) and model obtained by energy minimization in Chimera (yellow).

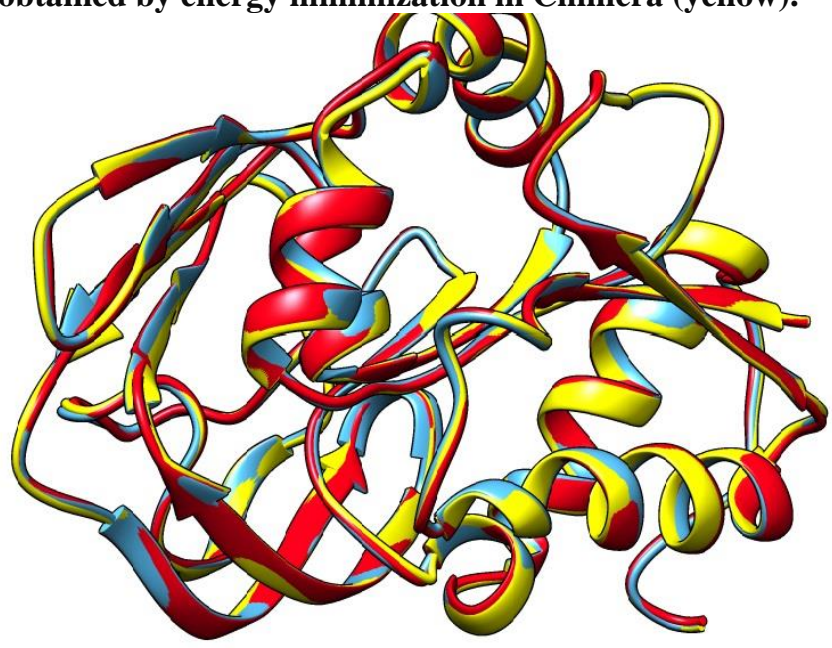

Figure 5. 3D image of pocket-1 of gatD as predicted by PrankWeb

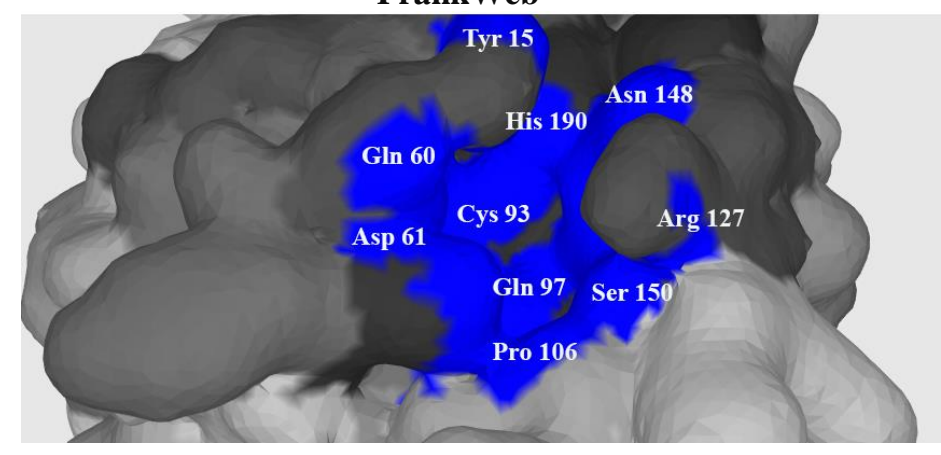

Protein structure prediction and validation

The 240 amino acids residue long gatD protein of $C$. botulinum was subjected to Swiss-model (https://swissmodel.expasy.org/) for comparative protein modelling. Comparative homology modelling depends on a sequence alignment between target sequence and the template sequence whose $3 \mathrm{D}$ structure has been determined by the experimental method. The results obtained from Swissmodelling server indicated that the target sequence is $52.7 \%$ identical to GatD from Streptococcus pneumonia (6FQB). GMQE (Global Model Quality Estimation) score is expressed as a number between 0 and 1 and reflects the expected accuracy of a model built with that alignment and template and the coverage of the target. A GMQE score of 0.77 was obtained which indicated higher reliability. The QMEAN Zscore provides an estimate of the "degree of nativeness" of the structural features observed in the model on a global scale. QMEAN Z-scores nearing zero indicate good agreement between the model structure and experimental structures of similar size. In the present investigation, we found that the QMEAN Z-score of gatD is -0.51 which is within the acceptable range, indicating a good quality model. The pairwise sequence alignment of gatD protein of C. botulinum (B1BAS5) and gatD protein Streptococcus pneumonia (Q8DNZ8) is shown in Figure 2. The homology model of gatD from $C$. botulinum was further subjected to energy minimization by using Chimera (version-1.13.1) and structural refinement by using 3-D refine (online server for protein structural refinement) [11]. The results of structural refinement through 3-D refine and energy minimization in Chimera are shown in Table 1. The validation for the generated models was done on the basis of Ramachandran plot, MolProbity Score, QMEAN Z-score, Errat Score and Verify 3D score. The MolProbity is a structure-validation web service that provides evaluation of protein and nucleic acid model quality at both the global and local levels [12]. Lower MolProbity scores are supposed to represent better quality models. We found that MolProbity scores of all models are in an acceptable range. The overall stereochemical quality of the 
Indo Global Journal of Pharmaceutical Sciences, 2020; 10(4): 46-57

modelled 3D structure of proteins was evaluated by using the Ramachandran plot which is based on psi ( $\mathrm{C} \alpha-\mathrm{C}$ bond) and phi (N-C $\alpha$ bond) angles of the protein and provides information about the number of amino acid residues present in allowed and disallowed regions. We found that the model generated by energy minimization and refinement by 3D refine web server consisted of 93.45 and $95 \%$ amino acid residues respectively in the favoured region, whereas the initial model shows 92.7 $\%$ of residues in the most favoured region (Figure 3, Table 1). Verify $3 \mathrm{D}$ determines the compatibility of an atomic model with its own amino acid sequence by assigning a structural class based on its location and environment and comparing the results to good structures. All the models generated in this study qualified the criterion used by Verify3D with more than $80 \%$ of the residues having averaged $3 \mathrm{D}-1 \mathrm{D}$ score $>=0.2$. The ERRAT scores of the models ranged from 88.8 to 97.3. Interestingly, the ERRAT score of the refined model was less when compared to the initial model and energy minimized one (Table 1). Overall the results indicated that models generated by energy minimization and refinement are more sophisticated and more conformationally better when compared to the initial model. The Figure4 shows the comparison of initial, refined model and energy minimized model in UCSF chimera.

Figure6. gatD ligand binding pocket - compound 1 interaction. A) 3D representation of interaction between compound 1 and residues in gatD ligand binding pocket. B) 2D interaction diagram of compound 1 with residues in gatD ligand binding pocket.

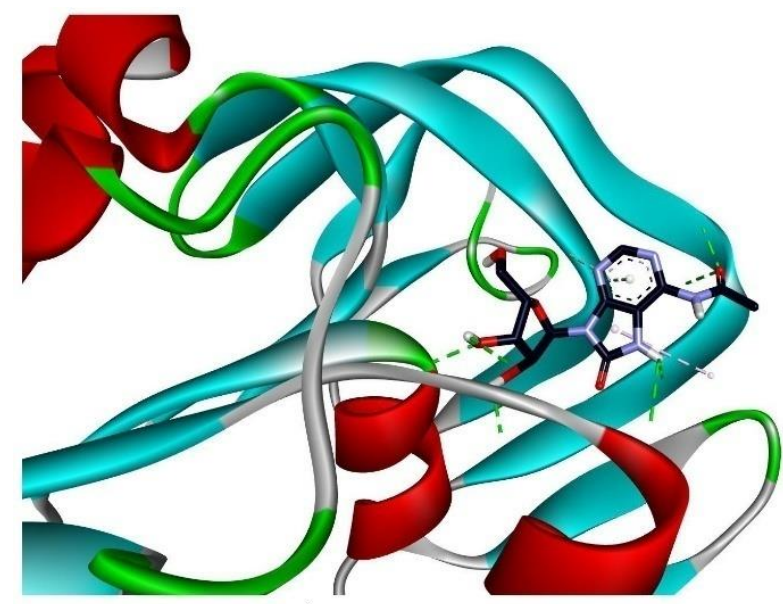

A

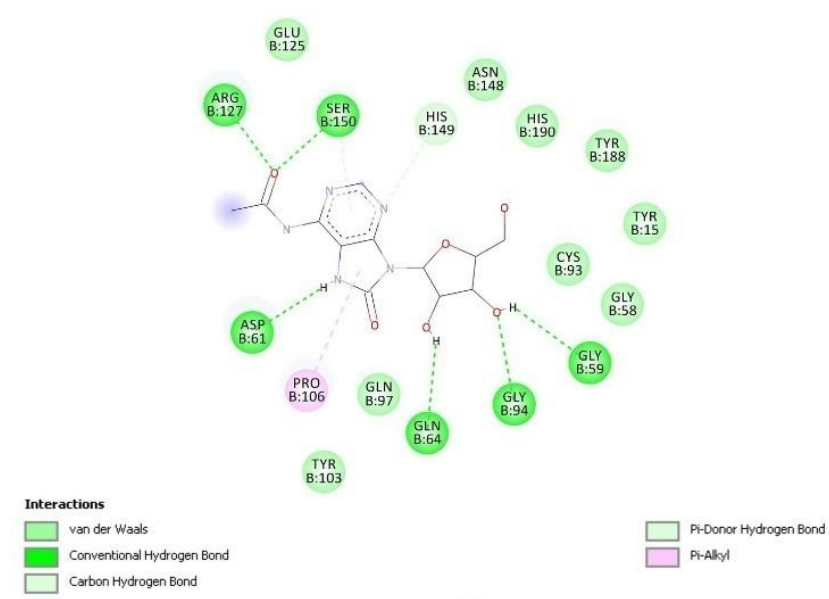

B

Figure7. gatD ligand binding pocket-Triciribine interaction. A) 3D representation of interaction between Triciribine and residues in gatD ligand binding pocket. B) 2D interaction diagram of Triciribine with residues in gatD ligand binding pocket.

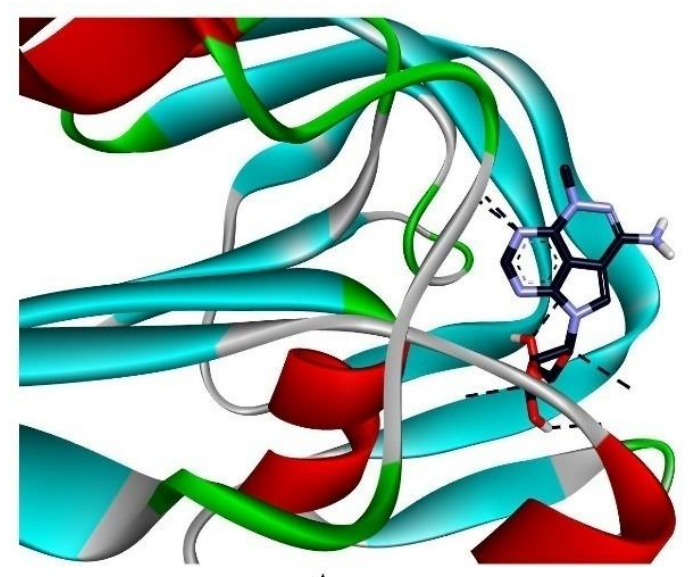

A

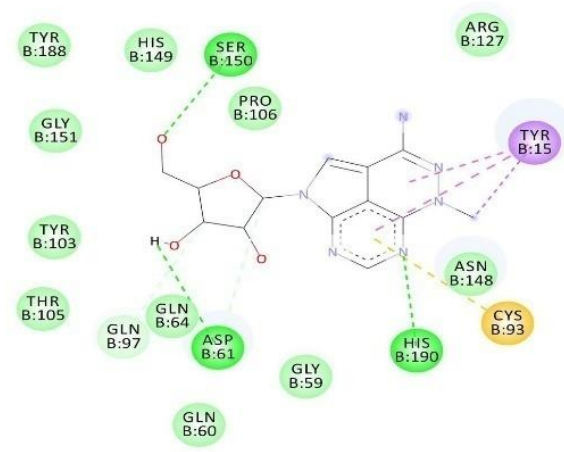

B 
Indo Global Journal of Pharmaceutical Sciences, 2020; 10(4): 46-57

Figure8. gatD ligand binding pocket-Iodotubercidin interaction. A) 3D representation of interaction between Iodotubercidin and residues in gatD ligand binding pocket. B) 2D interaction diagram of Iodotubercidin with residues in gatD ligand binding pocket.
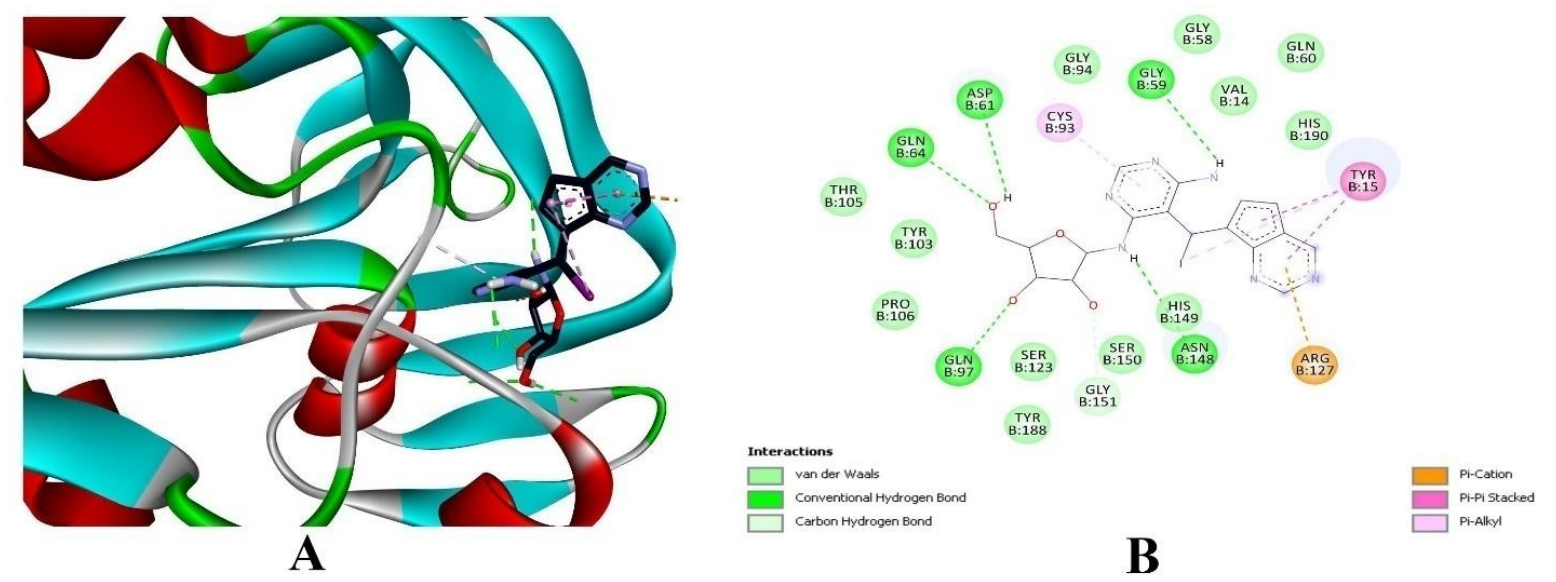

Figure9. gatD ligand binding pocket-Azasangivamycin interaction. A) 3D representation of interaction between Azasangivamycin and residues in gatD ligand binding pocket. B) 2D interaction diagram of Azasangivamycin with residues in gatD ligand binding pocket.
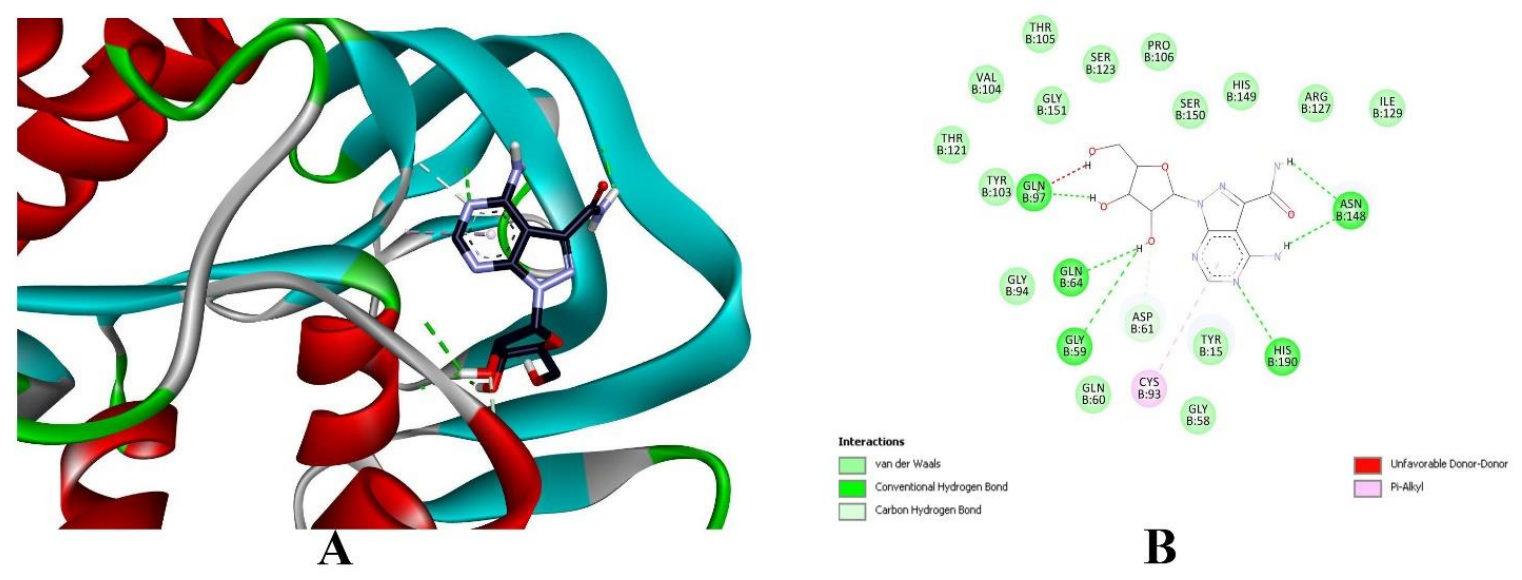

Figure 10. gatD ligand binding pocket-sangivamycin interaction. A) 3D representation of interaction between sangivamycin and residues in gatD ligand binding pocket. B) 2D interaction diagram of sangivamycin with residues in gatD ligand binding pocket

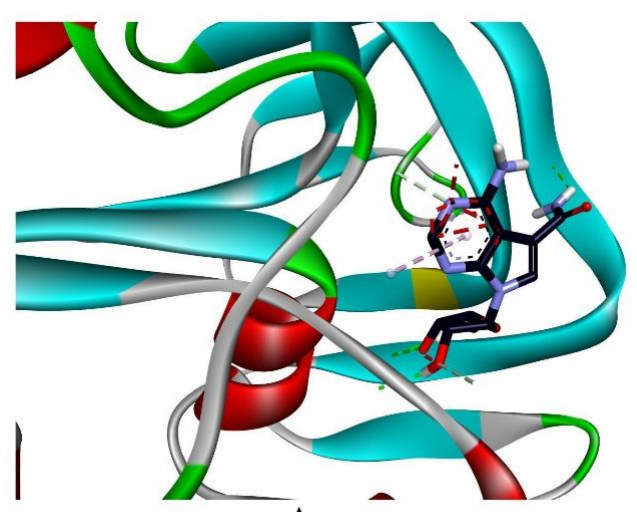

A

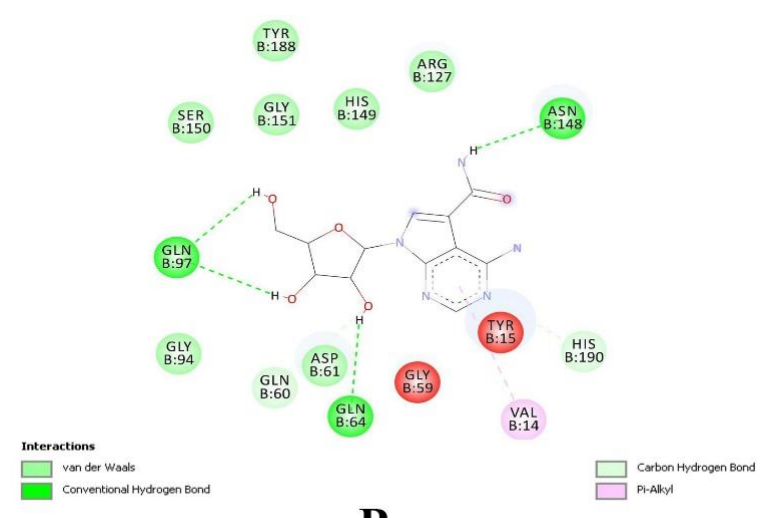

B 
Figure 11.gatD ligand binding pocket-Toyocamycin interaction. A) 3D representation of interaction between Toyocamycin and residues in gatD ligand binding pocket. B) 2D interaction diagram of Toyocamycin with residues in gatD ligand binding pocket.

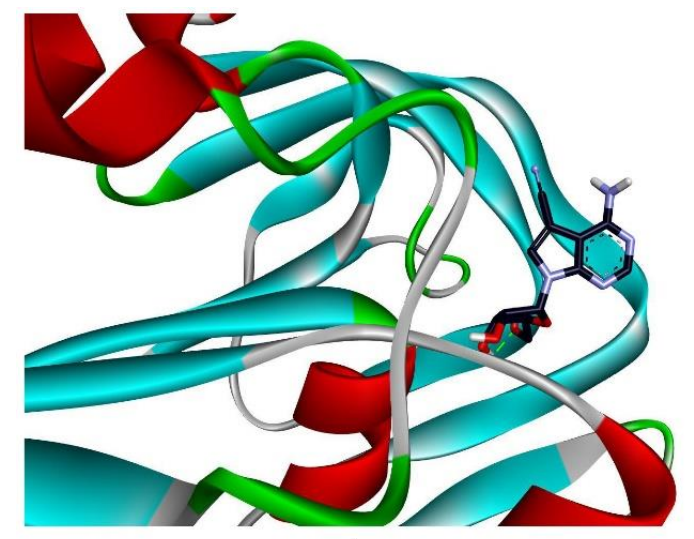

A

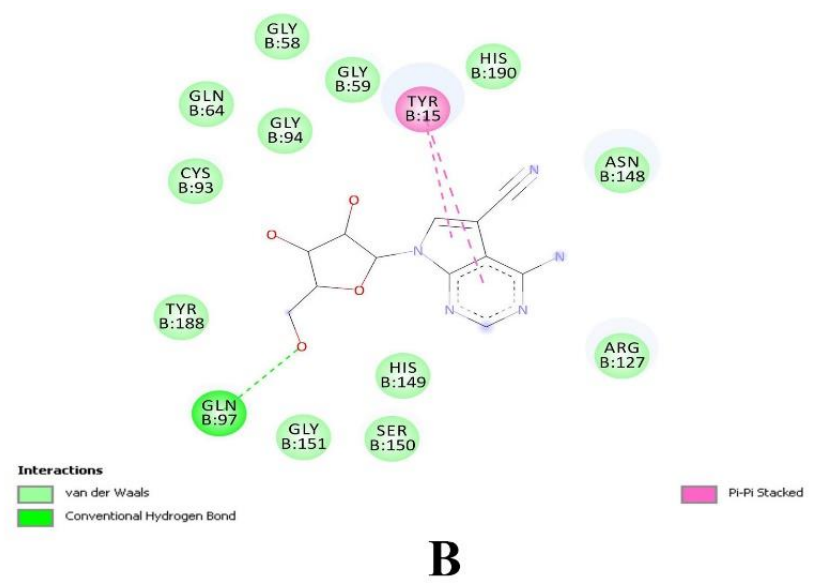

Figure 12.gatD ligand binding pocket-Tecadenoson interaction. A) 3D representation of interaction between Tecadenoson and residues in gatD ligand binding pocket. B) 2D interaction diagram of Tecadenoson with residues in gatD ligand binding pocket
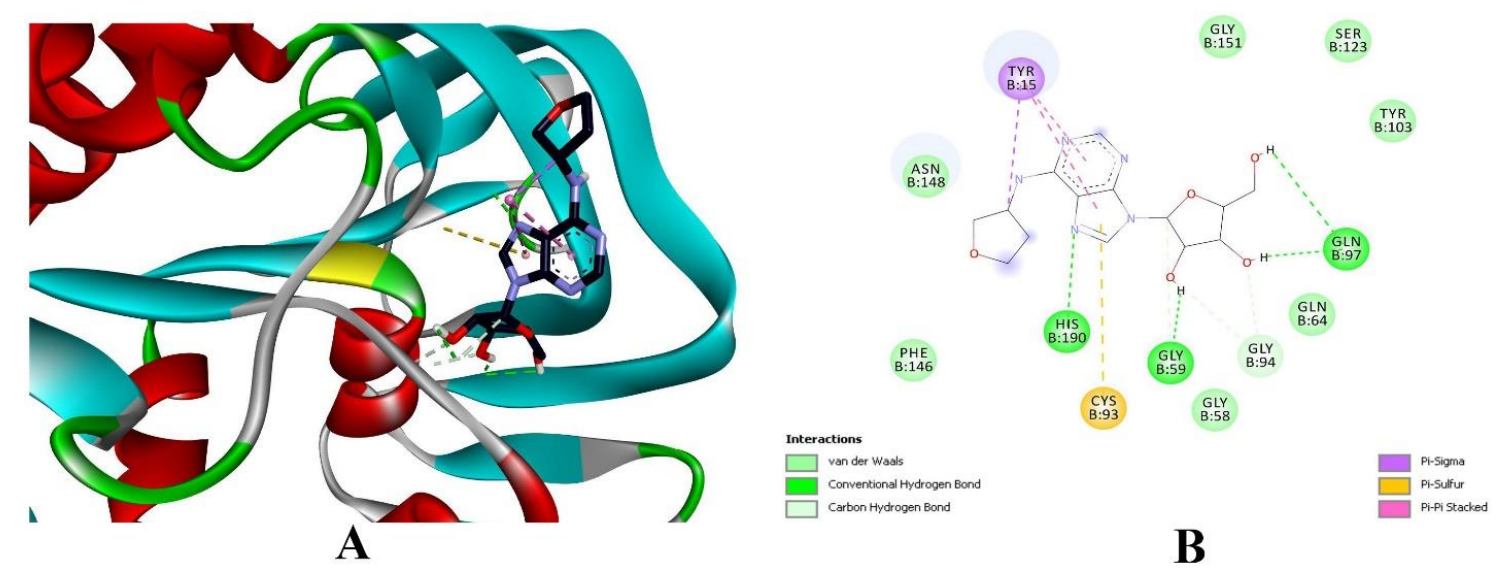

Figure 13.gatD ligand binding pocket-Compound 2 interaction. A) 3D representation of interaction between Compound 2 and residues in gatD ligand binding pocket. B) 2D interaction diagram of Compound 2 with residues in gatD ligand binding pocket.

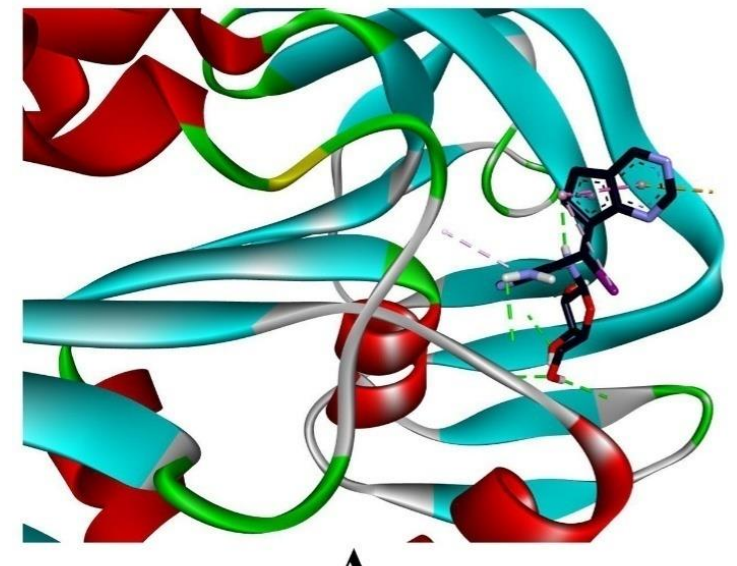

A

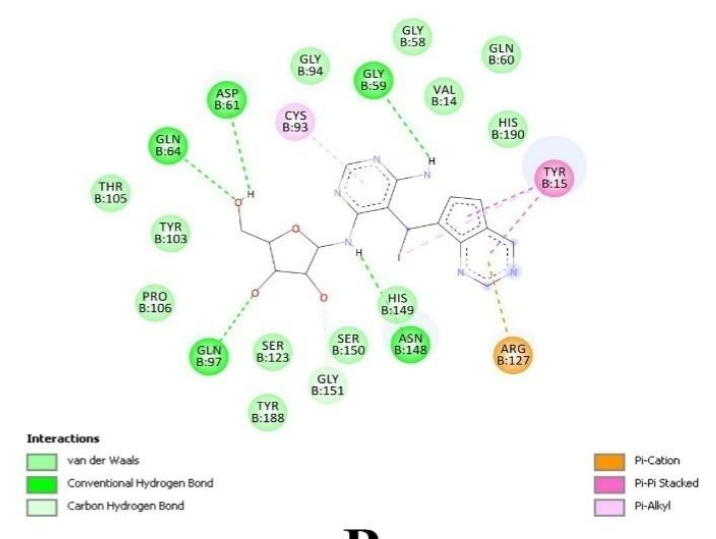

B 


\section{Indo Global Journal of Pharmaceutical Sciences, 2020; 10(4): 46-57}

\section{Ligand binding site prediction}

The ligand binding site of gatD was searched by using PrankWeb (http://prankweb.cz/). Three ligand binding pockets were predicted out of these the pocket-1 (with highest conservation score of 30.3) was selected (Table 2, Figure 5). The pocket- 1 is composed of a larger number of residues than any of the other pockets ( 21 amino acids). The 3D coordinate of pocket 1 is centred around $\mathrm{x}(-46.9701)$, y (38.9523) and $\mathrm{z}$ (36.7012) (Table 2).

\section{Molecular Docking of gatD with small molecules}

From the list of 1000 ligands obtained in SPOT-Ligand virtual screening, 30 compounds were subjected to molecular docking by using autodockvina. We further identified Triciribine phosphate (ZINC05385245) from the aforementioned list and subsequently docked Triciribine and related compounds (from Pubchem) with gatD. The list of compounds, molecular weight, and autodockvina binding energy are shown in Table 3. The autodock binding energy ranged from -7.1 to -8.6 . The molecular weight of the ligands ranged from 295 to 392. In autodockvina, the best binding energy was exhibited by compound $1(-8.6 \mathrm{kcal} / \mathrm{mol})$ followed by Triciribine $(-8.3$ $\mathrm{kcal} / \mathrm{mol})$. The ligand poses with the highest binding energy and $\mathrm{RMSD}=0$ were selected as the best pose. Discovery Studio 4.1 visualizer was employed to study the nature and types of interaction between residues of ligand binding pocket in gatD and compounds (Figure 6-13). We found that various non-covalent interactions viz. Hydrogen bond, Van der Waals interactions, Pi-alkyl interactions, alkyl interactions, Pi-cation interactions and $\mathrm{Pi}-\mathrm{Pi} \mathrm{T}$ shaped interactions between ligands and amino acid residues in the ligand binding pocket. The hydrogen bonds are a significant factor that contributes to the stability of protein-ligand binding interactions in molecular docking [13].The most frequent residues in the ligand binding pocket of gatD domain involved in hydrogen bond formation with ligands were Gly59, Gln64, His190 and Gln97. The maximum number of hydrogen bonds (6 numbers) were formed by compound 1 with the ligand binding pocket of gatD which correlates with high autodock vina binding energy (Table 3).Triciribine is a nucleoside analogue with a pentaazaacenaphthylene ring system linked with ribofuranosyl moiety by a glycosidic linkage (Table 4).Triciribine phosphate, a potent anti-cancer drug [14]and inhibitor of AKT (Protein Kinase B), is currently undergoing clinical trial against breast cancer (A study of PTX-200 (Triciribine) plus Cytarabine in refractory or relapsed Acute Leukemia. https://clinicaltrials.gov/ct2/show/NCT02930109, accessed on 3/1/2020). The compound 1 is related to triciribine but contain a ribofuranosyl moiety linked to a dihydropurine instead of pentaazaacenaphthylene ring (Table 4). The biological activity and target of the compound 1 are not yet identified. Iodotubericidin is an Iodopyrrolopyrimidine linked with ribofuranosyl moiety (Table 4). It is anti-tumour drug and strong p53 activator and used as an inhibitor for various kinases including adenosine kinase [15]. Sangivamycin, toyocomycin and compound 2 have pyrrolopyrimidine ring linked with ribofuranosyl moiety (Table 4).Sangivamycin is an anti-tumour agent and a potent inhibitor of protein kinase C [16] (Table 4). Toyocamycin is an $\mathrm{N}$-glycosylpyrrolopyrimidine in which the hydrogen at position 5 of the pyrrolopyrimidine moiety has been replaced by a cyano group (Table 4). Toyocamycin has antibiotic, antiviral as well as cytotoxic properties, and is known to inhibit the maturation of $40 \mathrm{~S}$ subunit of ribosome [17]. It acts as an antimetabolite, an antifungal agent, an antineoplastic agent, a bacterial metabolite and an apoptosis inducer. The biological activity and target for compound 2 is not yet identified. Tecadenoson is a selective A1 receptor agonist, which has been studied for their use against arrhythmia and atrial fibrillation [18]. Azasangivamycin is an analog of Sangivamycin in which the pyrrolopyrimidine ring is replaced by pyrazolopyrimidine. Azasangivamycin inhibits anti-viral properties and is active against Human cytomegalovirus and Herpes Simplex type I virus [19]. To conclude, except compound 1 and 2 all other compounds are reported as biologically active. gatD is an enzyme involved in amidation of $\alpha$-D-isoglutamicacid of cell wall precursor stem peptides in $S$. aureus. It has been demonstrated that $S$. aureus mutants defective in amidation are less viable and show increased susceptibility to antibiotics [2]. Therefore, inhibition of amidation reaction offers a novel strategy to combat $S$. aureus and other gram-positive bacterial pathogens such as $C$. botulinum. None of the compounds identified in this study were previously known as inhibitors of the gatD enzyme or amidation reaction. Therefore, we predict that all compounds identified via molecular docking are potential inhibitors of the gatD enzyme and amidation reaction. We further hypothesize that the compounds identified in this study can be developed as anti-amidation drugs in the near future. To the best of our knowledge, this is not only the first report of exploiting gatD as drug target but also prospective identification of antiamidation drugs. The compounds identified in this study can be used against infections caused by $C$. botulinum in the future. Further experimental studies are needed to support our hypothesis. 


\section{Indo Global Journal of Pharmaceutical Sciences, 2020; 10(4): 46-57}

\section{CONCLUSION}

The present study was undertaken for molecular and in silico characterization of gatD from $C$. botulinum. As the crystal structure for gatD was unavailable in the PDB databank, the strategy of homology modelling was utilized to generate its 3D structure. The generated model was refined and energy minimized and further validated to ensure better quality. The phylogenetic analysis revealed the presence of three distinct clusters and highlighted series of duplication and diversification events in each cluster. The ligand binding pockets in gatD and the associated residues were identified using PrankWeb. Further, this study is first of its kind to predict the molecular mechanism(s) underlying the docking of series of anti-amidation agent to the target enzyme, gatD, indicating the tremendous potential of these agents in hindering the growth of $C$. botulinum and other related species. As the majority of these compounds are also known as anti-cancer and antiviral agents, they are worth of further experimental investigations in terms of inhibition of cell wall synthesis, amidation and anti-microbial properties. The findings of this study are expected to have an impact on the current emergence of research focus on the novel speciesspecific antimicrobial agents by investigating the unexplored aspects of the molecular nature of its interaction with target enzyme, gatD.

\section{ACKNOWLEDGEMENT}

The authors are extremely grateful to Dr. Anisha GS, Department of Zoology, and Dr. Sunil Kesava Deth G, Department of Botany, Government College for Women, Thiruvananthapuram, Kerala for providing necessary facilities.

\section{CONFLICTS OF INTEREST}

None.

\section{DATA AVAILABILITY}

The data used to support the findings of this study are included within the article.

\section{FUNDING SOURCE}

Funding under the DST-FIST scheme to Government College for Women, Thiruvananthapuram is duly acknowledged

\section{REFERENCES}

[1] Van Heijenoort, J. Lipid Intermediates in the Biosynthesis of Bacterial Peptidoglycan.Microbiol. Mol. Biol. Rev., 2007; 71(4):620635.

[2] Nöldeke, E.R. et al. Structural basis of cell wall peptidoglycan amidation by the GatD/MurT complex of Staphylococcus aureus. Scientific Reports., 2018; 8(1):1-15.
[3] Gotz, F., Bannerman, T.,Schleifer, K.H. The Genera Staphylococcus and Macrococcus.Prokaryotes. In: A Handbook on the Biology of Bacteria, $3^{\text {rd }}$ d; Springer, 2006; 4: 45-75

[4] Münch, D., Roemer, T., Lee, S.H., Engeser, M., Sahl,HG., Schneider, T. Identification and in vitro analysis of the GatD/MurT enzyme-complex catalyzing lipid II amidation in Staphylococcus aureus. PLoS Pathogens., 2012; 8(1).

[5] Carter, A.T., Peck, M.W. Genomes, neurotoxins and biology of Clostridium botulinum Group I and Group II. Research in Microbiology., 2015; 166(4):303-17.

[6] Mazuet, C., Legeay, C., Sautereau, J., Ma, L., Bouchier, C., Bouvet, P.,Popoff, M.R. Diversity of group I and II Clostridium botulinum strains from France including recently identified subtypes: Genome Biology and Evolution., 2016; 8:1643-60.

[7] Fenicia, L., Anniballi, F. Infant botulism. Ann.Ist. Super.Sanita., 2009;45(2):134-146.

[8] Katsila, T., Spyroulias, G.A., Patrinos, G.P.,Matsoukas, M.T. Computational approaches in target identification and drug discovery. Computational and Structural Biotechnology Journal., 2016; 14:177184

[9] Kumar, S., Stecher, G., Tamura, K. MEGA7: Molecular Evolutionary Genetics Analysis Version 7.0 for Bigger Datasets., Molecular biology and evolution., 2016; 33:1870-74.

[10] Litfin, T., Zhou, Y., Yang Y. SPOT-ligand 2.improving structure-based virtual screening by binding-homology search on an expanded structural template library. Bioinformatics., 2017; 33:12381240 .

[11] Bhattacharya, D., Nowotny,.J, Cao, R., Cheng, J. 3Drefine: an interactive web server for efficient protein structure refinement. Nucleic acids research., 2016; 44:406-9.

[12] Chen, V.B. et al. MolProbity: All-atom structure validation for macromolecular crystallography.ActaCrystallographica., 2010; 66(1) $12-21$.

[13] Patil, R., Das, S., Stanley, A., Yadav, L., Sudhakar, A.,Varma, A.K. Optimized hydrophobic interactions and hydrogen bonding at the target-ligand interface leads the pathways of drug-designing. Plos One., 2010; 5:1

[14] Garrett, C.R. et al. Phase I pharmacokinetic and pharmacodynamic study of Triciribine phosphate monohydrate, a small-molecule inhibitor of AKT phosphorylation, in adult subjects with solid tumors containing activated AKT. Investigational New Drugs., 2011; 29:1381-9.

[15] Zhang, X. et al. Identification of 5-Iodotubercidin as a Genotoxic Drug with Anti-Cancer Potential. PLoS One., 2013; 8(5)

[16] Loomis, C.R., Bell, R.M. Sangivamycin, a nucleoside analogue, is a potent inhibitor of protein kinase C. Journal of Biological Chemistry., 1988; 263:1682-1692.

[17] Kiburu, I.N., LaRonde-LeBlanc, N. Interaction of Rio1 Kinase with Toyocamycin Reveals a Conformational Switch That Controls Oligomeric State and Catalytic Activity. PLoS One., 2012; 7(5)

[18] Rabuffetti, M., Bavaro, T., Semproli.R., Cattaneo, G., Massone, M., Morelli, C.F.Synthesis of Ribavirin, Tecadenoson, and Cladribine by Enzymatic Transglycosylation. Catalysts., 2019; 9(4):355.

[19]Migawa, M.T., Drach, J.C., Townsend, L.B.Design, Synthesis and Antiviral Activity of Novel 4,5-Disubstituted 7-( $\beta-\mathrm{d}$ Ribofuranosyl)pyrrolo[2,3- $d$ ][1,2,3]triazines and the Novel 3Amino-5-methyl-1-( $\beta$ - $d$-ribofuranosyl)- and 3-Amino-5-methyl-1- 
Indo Global Journal of Pharmaceutical Sciences, 2020; 10(4): 46-57

(2-deoxy- $\beta$ - $\quad$ d $\quad$-ribofuranosyl)-1,5-dihydro-1,4,5,6,7,8-

hexaazaacenaphthylene as Analogues of Triciribine Journal of

Medicinal Chemistry., 2005; 48:3840-3851.

Indo Global Journal of Pharmaceutical Sciences( ISSN 2249 1023; CODEN- IGJPAI; NLM ID: 101610675) indexed and abstracted in CrossRef (DOI Enabling), CNKI, UGC CARE Journal List, EMBASE (Elsevier), National Library of Medicine (NLM) Catalog (NCBI), ResearchGate, Publons (Clarivate Analytics), CAS (ACS), Index Copernicus, Google Scholar and many more. For further details, visit http://iglobaljournal.com 